\title{
Primary cutaneous aspergillosis and idiopathic bone marrow aplasia*
}

\author{
Karina Colossi Furlan ${ }^{1}$ \\ Priscila Kakizaki ${ }^{1}$ \\ Neusa Yuriko Sakai Valente ${ }^{1}$
}

\author{
Mario Cezar Pires ${ }^{2}$ \\ Juliana Cabral Nunes Chartuni ${ }^{3}$
}

DOI: http:/ /dx.doi.org/10.1590/abd1806-4841.20164889

\begin{abstract}
We describe the case of a 9-year-old boy with idiopathic bone marrow aplasia and severe neutropenia, who developed skin ulcers under cardiac monitoring electrodes. The diagnosis of primary cutaneous aspergillosis was made after the second biopsy and culture. Imaging investigation did not reveal internal fungal infection. The child was treated, but did not improve and died 3 months after admission. The report highlights and discusses the preventable risk of aspergillus skin infection in immunocompromised patients.
\end{abstract}

Keywords: Aspergillosis; Bone marrow; Immunosuppression; Spores, fungal

\section{INTRODUCTION}

Aspergillus spp. is the second most common cause of opportunistic fungal infection in humans after Candida albicans. ${ }^{1}$ It causes severe infections in immunocompromised patients, resulting in high mortality, specially in neonates. ${ }^{2}$ The usual infection organs are the lungs, central nervous system (CNS) and paranasal sinuses.

Primary cutaneous aspergillosis (PCA) is a rare condition, usually associated with immunodeficiency, secondary to hematologic disorders. ${ }^{1}$ There are two different inoculation routes into the skin: non-sterile devices or airborne Aspergillus conidia colonization of the traumatized skin. ${ }^{3}$ Herein, we evidence the first case of PCA under the cardiac monitoring electrodes, in a child with idiopathic bone marrow aplasia.

\section{CASE REPORT}

A 9-year-old boy diagnosed with idiopathic bone marrow aplasia was admitted to the pediatric intensive care unit (ICU) with septic shock and respiratory insufficiency, requiring mechanical ventilation and cardiac monitoring. He suffered from severe neutropenia and after a few weeks, developed a fever of unknown origin (FUO). The patient received broad-spectrum antibiotic and antifungal therapy, without clinical improvement.

After 45 days on the pediatric ICU, the patient developed circular erythematous lesions with a necrotic center under the cardiac monitoring electrodes (Figure 1). The lesions underwent extensive necrosis in 10 days (Figure 2). The second biopsy performed

Received on 29.06.2015

Approved by the Advisory Board and accepted for publication on 05.09.2015

Work performed at the Hospital do Servidor Público Estadual (HSPE) - São Paulo (SP), Brazil.

Financial Support: None.

Conflict of Interest: None.

Hospital Público Estadual do Estado de São Paulo (HSPE) - São Paulo (SP), Brazil

Complexo Hospital de Padre Bento de Guarulhos - Guarulhos (SP), Brazil.

Hospital Regional de Santa Maria - Brasília (DF), Brazil

C2016 by Anais Brasileiros de Dermatologia 
showed septate hyaline hyphae upon hematoxylin-eosin staining (Figure 3). Grocott staining showed many septate hyphae with branches at acute angles (Figure 4). Culture of the material in Sabouraud dextrose agar plate revealed darker reverse coloration, while microcultive in lamina technique confirmed the presence of the Aspergillus niger species (Figure 5). A whole body computed tomography scan did not reveal disseminated fungal infection.

The patient was already receiving amphotericin $\mathrm{B}$ and fluconazole. After the diagnosis, voriconazole was added to antifungal therapy, though there was no improvement and the patient died 3 months after admission.

\section{DISCUSSION}

Cutaneous aspergillosis can be either primary or secondary. Primary cutaneous lesions usually result from direct inoculation of the Aspergillus species due to trauma, especially in patients on catheter care, trauma from arm boards, burns and infected dressings. There are also reported cases of fungi in aerosol at neonatal ICUs during building renovation. ${ }^{1}$ Secondary cutaneous aspergillosis stems from disseminated infection. ${ }^{1}$

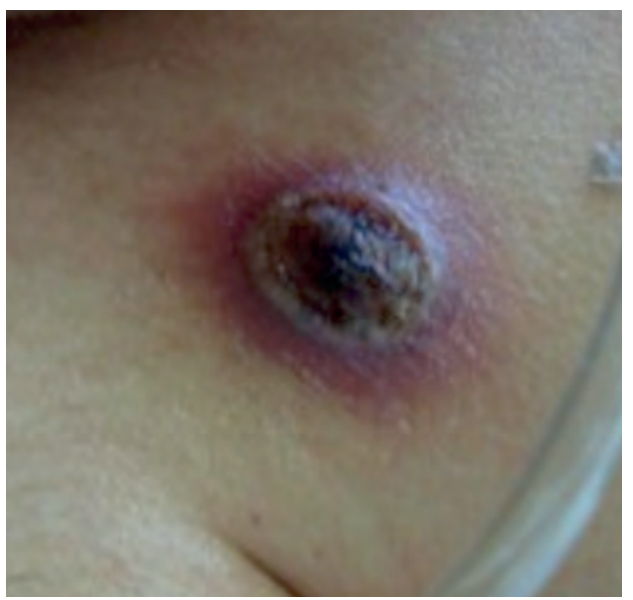

Figure 1 :

Erythematous circular lesion with a necrotic center under the cardiac monitoring electrode

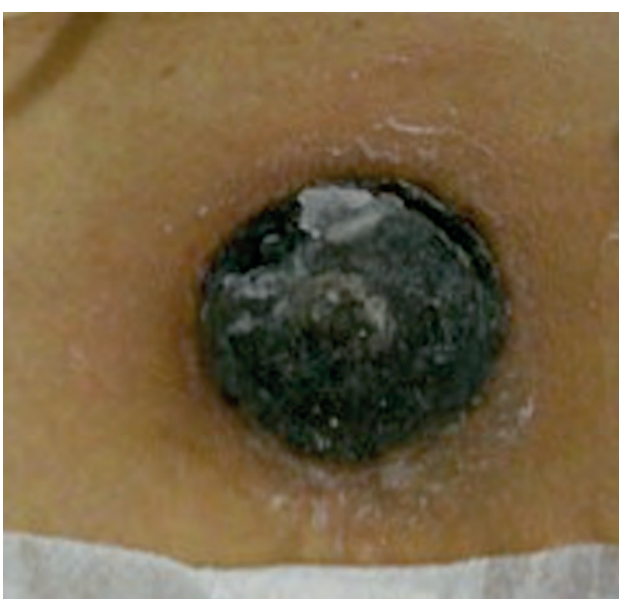

Figure 2 :

$\mathrm{Necrotic}$ c i r c u l a r lesion under the cardiac monitoring electrode

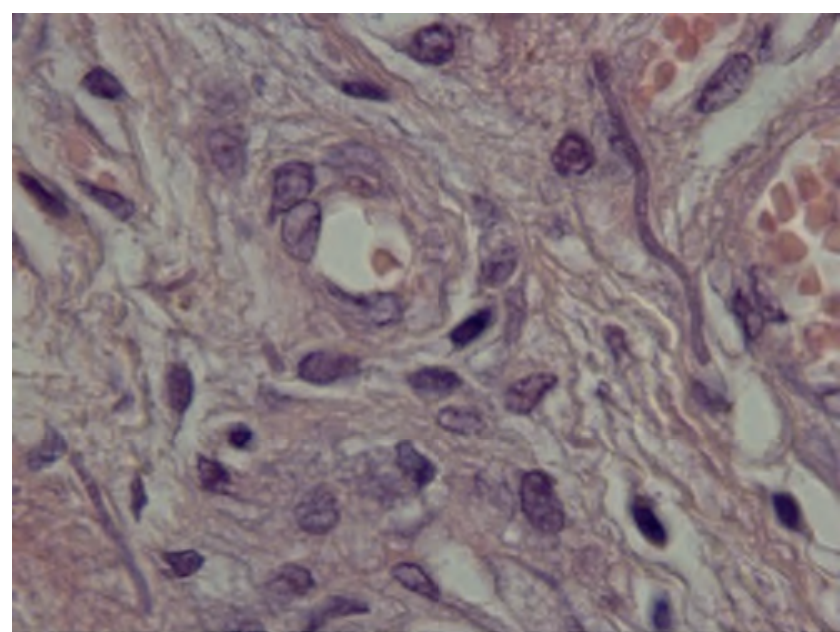

FIGURE 3 : Septate hyaline hyphae on tissue sample (HematoxylinEosin staining)

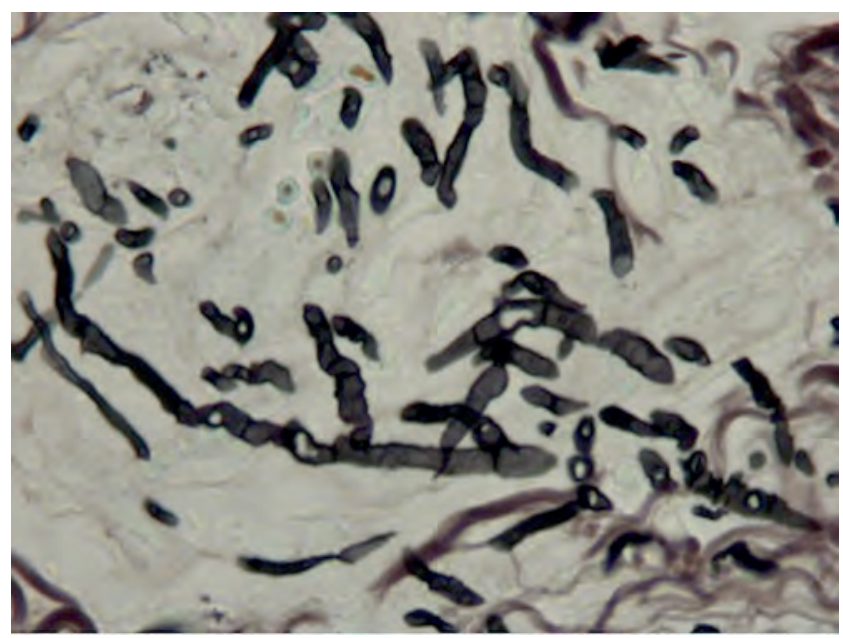

FIGURE 4 : Grocott staining on tissue sample shows many septate hyphae with branches at acute angles

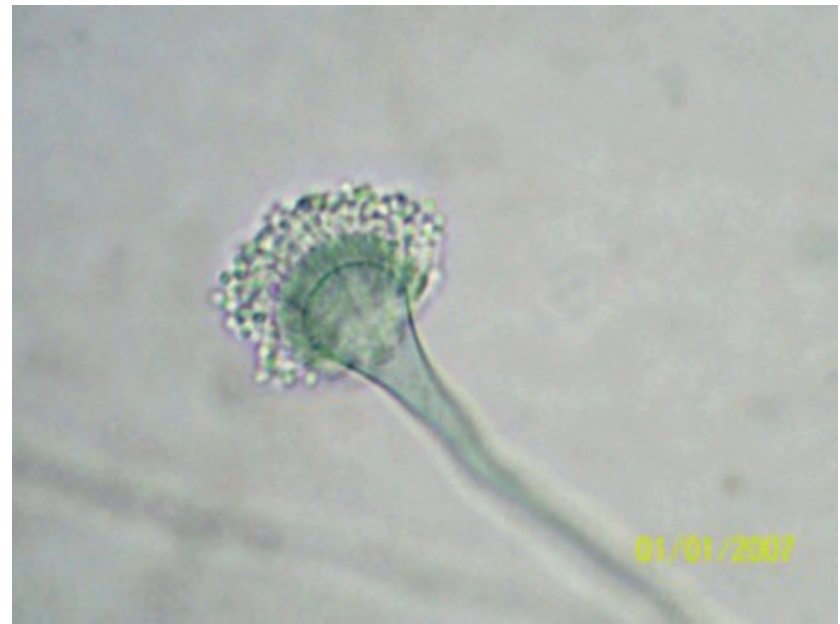

Figure 5 : Microcultive in lamina confirmed the species Aspergillus niger 
PCA is usually caused by Aspergillus flavus, A. terreus, A. niger and A. ustus. The lesion may present as erythematous, indurated macules, papules, plaques or hemorrhagic bullae, which can progress to necrotic ulcers that are covered by black eschar. Although rare, nodules and pustular lesions can also be present. ${ }^{4,5}$

Tahir et al. ${ }^{1}$ described an immunocompetent female with multiple ulcers in the axillae and perineum. She most probably contracted the infection from contaminated palm oil on which she was conducting a research and got inoculated after shaving her axillae and perineum with razor blades. The patient fully recovered after wound surgery. ${ }^{1}$ Anderson et al. ${ }^{3}$ described a child diagnosed with acute myeloid leukemia, who developed two asymptomatic, geometric, erythematous dermal plaques on his right forearm. The skin lesions occurred at the site of tapes applied to secure an arm board for intravenous access and the culture isolated Aspergillus niger. The eruption rapidly resolved with systemic anti-fungal therapy. ${ }^{3}$ Stock et al. reported necrotic skin lesions in the back, perineum, and axillae in a premature neonate. ${ }^{6}$ Aspergillus fumigatus was isolated and a disposable, non-sterile glove was found to be contaminated with Aspergillus fumigatus. This glove could have infected the neonate's skin. ${ }^{6}$ The routes of inoculation in our patient include the use of non-sterile cardiac monitoring electrodes, harboring aspergillus on the skin.

\section{REFERENCES}

1. Tahir C, Garbati M, Nggada HA, Yawe EH, Abubakar AM. Primary cutaneous aspergillosis in an immunocompetent patient. J Surg Tech Case Rep. 2011;3:94-6.

2. Andresen J, Nygaard EA, Størdal K. Primary cutaneous aspergillosis (PCA)-a case report. Acta Paediatr. 2005;94:761-2.

3. Anderson A, Foster RS, Brand R, Blyth CC, Kotecha RS.Acute Onset of Pustules at the Site of Tape Placement in an Immunocompromised Infant with Acute Myeloid Leukemia. Pediatr Dermatol. 2014;31:609-10.

4. Ajith C, Dogra S, Radotra BD, Chakrabarti A, Kumar B. Primary cutaneous aspergillosis in an immunocompetent individual. J Eur Acad Dermatol Venereol. 2006;20:738-9.

5. Ozer B, Kalaci A, Duran N, Dogramaci Y, Yanat AN. Cutaneous infection caused by Aspergillus terreus. J Med Microbiol. 2009;58:968-70.

6. Stock C, Veyrier M, Raberin H, Fascia P, Rayet I, Lavocat MP, et al. Severe cutaneous aspergillosis in a premature neonate linked to nonsterile disposable glove contamination? Am J Infect Control. 2012;40:465-7

7. Saghrouni F, Ben Youssef Y, Gheith S, Bouabid Z, Ben Abdeljelil J, Khammari I, et al. Twenty-nine cases of invasive aspergillosis in neutropenic patients. Med Mal Infect. 2011;41:657-62.

8. Walsh TJ, Anaissie EJ, Denning DW, Herbrecht R, Kontoyiannis DP, Marr KA, et al. Treatment of Aspergillosis: Clinical Practice Guidelines of the Infectious Diseases Society of America. Clin Infect Dis. 2008;46:327-60.
The diagnosis of PCA is carried out with a biopsy and culture. In some cases, hyphae can be observed upon mycological examination under direct microscopic examination. ${ }^{7}$

The treatment of aspergillosis is made with systemic antifungal drugs like amphotericin $B$ and itraconazole. Treatment of primary cutaneous fungal infection is controversial; both medical and surgical modalities have been used. ${ }^{8}$ Voriconazole and deoxycholate amphotericin B are the only licensed drugs in the United States for primary treatment of invasive aspergillosis. Itraconazole and caspofungin have been approved for salvage therapy of invasive aspergillosis. ${ }^{8}$

In immunocompromised patients with unusual skin lesions, we should always suspect infectious disease. Biopsy and cultures are essential for pathogen identification and more than one sample may be required for correct diagnosis. We strongly recommend the use of sterile disposable devices in immunocompromised patients. Our case highlights the preventable risk of PCA and the importance of closely monitoring the evolution of skin changes associated with the use of medical devices.]

\author{
MAILING ADDRESS: \\ Karina Colossi Furlan \\ Av. Ibirapuera, 981 \\ Indianópolis \\ 04028-000 - São Paulo - SP \\ Brazil \\ E-mail:kcolossi@gmail.com
}

How to cite this article: Furlan KC, Pires MC, Kakizaki P, Chartuni JCN, Valente NYS. Primary cutaneous aspergillosis and idiopathic bone marrow aplasia. An Bras Dermatol. 2016;91(3):381-3. 